\title{
PERIODIC AND FIXED POINTS, AND COMMUTING MAPPINGS
}

\author{
GERALD JUNGCK
}

\begin{abstract}
We employ commuting mappings to produce generalizations of locally contractive and locally expansive maps, and obtain criteria for the existence of fixed and periodic points of arbitrary maps on compacta.
\end{abstract}

1. Introduction. Our main result cites conditions which ensure that a map of a compact metric space onto itself has periodic points. This one theorem yields as by-products a necessary and sufficient criterion for the existence of fixed points (Corollary 2.3), a generalization of results of Bailey [1] and Holmes [3] on locally contractive maps, and a theorem on periodic points for open and generalized locally expansive maps.

By the term map we shall mean a continuous function. A map $f:(X, d) \rightarrow$ $(X, d)$ is locally contractive (expansive) iff there exists $\varepsilon>0$ such that $d(f(x), f(y))<d(x, y)(>d(x, y))$ whenever $0<d(x, y)<\varepsilon$. Maps $f, g$ : $X \rightarrow X$ commute iff $f g=g f$. If $g: X \rightarrow X$, we let $C_{g}$ denote the set of all maps $f: X \rightarrow X$ which commute with $g$. In addition, $N$ denotes the set of natural numbers, and for each $n \in N, f^{n}$ denotes the $n$th composite of $f$.

The fact that a function $f: X \rightarrow X$ has fixed points iff there is a constant function $g: X \rightarrow X$ which commutes with $f$ prompts the investigation of commuting mappings in the search for fixed points (see [4]). Their potential as a means of generalizing lies in the fact that, for any $n, f^{n} \in C_{g}$ if $f \in C_{g}$ (in particular, $g^{n} \in C_{g}$ ).

\section{Main result and corollaries.}

TheOREM 2.1. Let $g$ be a surjective map of a compact metric space $(X, d)$ to itself. Suppose $\varepsilon$ is a positive number satisfying the condition: if $x, y \in X$ and $0<d(g(x), g(y))<\varepsilon$ then there is at least one $f \in C_{g}$ and $z \in g^{-1}(g(x))$ such that

$$
d(f(z), f(y))<d(g(x), g(y)) .
$$

Then $\mathbf{g}$ has a periodic point. In fact, if $k$ is a positive integer such that, for some $x, d\left(x, g^{k}(x)\right)<\varepsilon$, then $a=g^{k}(a)$ for some $a \in X$.

Received by the editors August 19, 1978 and, in revised form, November 7, 1978.

AMS (MOS) subject classifications (1970). Primary 54E40; Secondary 54H25.

Key words and phrases. Locally contractive and locally expansive maps, commuting maps, periodic points, and fixed points. 
Proof. Let $x \in X$. The sequence $\left\{g^{n}(x)\right\}$ has a convergent subsequence since $(X, d)$ is compact. Specifically, there exist $k$ and $m$ such that

$$
d\left(g^{m}(x), g^{m+k}(x)\right)=d\left(g^{m}(x), g^{k}\left(g^{m}(x)\right)\right)<\varepsilon .
$$

Thus:

(i) $d\left(y, g^{k}(y)\right)<\varepsilon$ for some $y \in X$.

Suppose that $k \in N$ for which (i) holds. Since the composite $g^{k}: X \rightarrow X$ is continuous and $(X, d)$ is compact, there exists $a \in X$ for which

(ii) $d\left(a, g^{k}(a)\right)<d\left(x, g^{k}(x)\right)$ for all $x \in X$.

We assert that $a=g^{k}(a)$. Otherwise, (i) implies $0<d\left(a, g^{k}(a)\right)<\varepsilon$. But $g$ is a surjection, so that $g(c)=a$ for some $c \in X$, and we have

$$
0<d\left(g(c), g^{k}(g(c))\right)=d\left(g(c), g\left(g^{k}(c)\right)\right)<\varepsilon .
$$

Consequently, the hypothesis yields $f \in C_{g}$ and $z \in g^{-1}(g(c))$ such that

$$
d\left(f(z), f\left(g^{k}(c)\right)\right)<d\left(g(c), g\left(g^{k}(c)\right)\right) \text {. }
$$

Since $z \in g^{-1}(g(c)), g^{k}(z)=g^{k}(c)$, and hence

$$
d\left(f(z), f\left(g^{k}(z)\right)\right)<d\left(g(c), g\left(g^{k}(c)\right)\right) .
$$

But then

$$
d\left(f(z), g^{k}(f(z))\right)<d\left(a, g^{k}(a)\right)
$$

since $f \in C_{g}$. This last inequality contradicts (ii).

Now let $g$ be any map (not necessarily surjective) of a compact metric space $X$ to itself. If $A=\bigcap_{n=1}^{\infty} g^{n}(X)$, then $A$ is compact, $g(A)=A$, and if $f \in C_{g}$, then $\left.\left.f\right|_{A} \in C_{g}\right|_{A}$. Thus, if we require that the $z \in g^{-1}(g(x))$ in Theorem 2.1 be $x$, we can apply 2.1 to $\left.g\right|_{A}: A \rightarrow A$ to conclude:

COROllary 2.2. Let $g$ be a map of a compact metric space $(X, d)$ to itself. If $\varepsilon$ is a positive number such that whenever $0<d(g(x), g(y))<\varepsilon$, there exists $f \in C_{\mathbf{g}}$ for which

$$
d(f(x), f(y))<d(g(x), g(y))
$$

then $\mathrm{g}$ has a periodic point. In fact, if $k$ is a positive integer such that, for some $x \in A=\bigcap_{n=1}^{\infty} g^{n}(X), d\left(x, g^{k}(x)\right)<\varepsilon$, then $a=g^{k}(a)$ for some $a \in X$.

The following fixed point theorem is stated without proof in [4].

Corollary 2.3. A map $g$ of a compact metric space $(X, d)$ into itself has a fixed point iff $g(x) \neq g(y)$ implies there is some $f \in C_{8}$ such that $d(f(x), f(y))$ $<d(g(x), g(y))$.

Proof. The necessity portion is easily proved by considering the constant function $f(x) \equiv a$, where $a$ is a fixed point of $g$ (see the proof of the theorem in [4]). To prove "sufficiency", let $\varepsilon=2 \operatorname{diam}(X)$, note that $d(x, g(x))<\varepsilon$ for all $x \in X$, and appeal to Corollary 2.2. 


\section{Locally contractive maps generalized.}

THEOREM 3.1. Let $H$ be a commutative semigroup of maps of a compact metric space $(X, d)$ to itself and let $g \in H$. If $\varepsilon$ is a positive number such that whenever $0<d(g(x), g(y))<\varepsilon$, there is some $h \in H$ satisfying

$$
d(h(x), h(y))<d(g(x), g(y)),
$$

then $g$ and any finite subfamily of $H$ have a common periodic point.

Proof. For $h \in H$ and $k \in N$ let $P(h, k)=\left\{x \in X: h^{k}(x)=x\right\}$. These sets are closed since $h$ is continuous. Note also that $f(P(h, k)) \subset P(h, k)$ if $f \in H$, since $f$ and $h$ commute. By Corollary 2.2 there exists $m$ such that $P(g, m) \neq \varnothing$. Denote $P(g, m)$ by $P_{g}$, and consider:

LEMma. Assume the hypothesis of Theorem 3.1, and let $F$ be a nonempty closed subset of $P_{g}$ such that $h(F) \subset F$ for all $h \in H$. If $h \in H, h$ has a periodic point in $F$.

Proof. Since $P_{g}$ is closed, $(F, d)$ is compact. Let $h \in H$ and apply the initial argument of the proof of Theorem 2.1 to $\left.h\right|_{F}$ to produce $a \in F$ and $k \in N$ such that $d\left(a, h^{k}(a)\right)<\varepsilon$ and

$$
d\left(a, h^{k}(a)\right)<d\left(x, h^{k}(x)\right) \text { for } x \in F .
$$

Suppose $a \neq h^{k}(a)$. Since $a \in P_{g}, g^{m}(a)=a$. Let $c=g^{m-1}(a)$. Then

$$
d\left(h^{k}(a), a\right)=d\left(h^{k}(g(c)), g(c)\right)=d\left(g\left(h^{k}(c)\right), g(c)\right) \text {. }
$$

Thus $0<d\left(g\left(h^{k}(c)\right), g(c)\right)<\varepsilon$, so by hypothesis there exists $f \in H$ such that

$$
d\left(g\left(h^{k}(c)\right), g(c)\right)>d\left(f\left(h^{k}(c)\right), f(c)\right)
$$

i.e., $d\left(h^{k}(a), a\right)>d\left(h^{k}(f(c)), f(c)\right)$. But $f(c) \in F$ since $a \in F$ and hence $c \in$ $F$; consequently, the last inequality above denies (*). Conclude that $a=$ $h^{k}(a)$.

We now complete the proof of Theorem 3.1. Observe that $P_{g}$ is a nonempty closed subset of $P_{g}$ such that $h\left(P_{g}\right) \subset P_{g}$ for $h \in H$. The Lemma therefore yields $k=k(h)$ for each $h \in H$ such that $P_{g} \cap P(h, k) \neq \varnothing$. Thus any singleton subset of $H$ has a periodic point in common with $g$. We proceed by induction.

Let $\left\{h_{1}, h_{2}, \ldots, h_{n}, h_{n+1}\right\} \subset H$ and assume that

$$
G_{n}=P_{g} \cap\left(\bigcap_{i=1}^{n} P\left(h_{i}, k_{i}\right)\right) \neq \varnothing .
$$

Since $f(P(h, k)) \subset P(h, k)$ for any $f, h \in H$ and $k \in N$,

$$
f\left(G_{n}\right) \subset f\left(P_{g}\right) \cap\left(\bigcap_{i=1}^{n} f\left(P\left(h_{i}, k_{i}\right)\right)\right) \subset G_{n} \text { for any } f \in H .
$$

Applying the Lemma to $h_{n+1}$ and $G_{n}$ guarantees the existence of a $k_{n+1} \in N$ such that $G_{n} \cap P\left(h_{n+1}, k_{n+1}\right) \neq \varnothing$. Thus, by induction, $g$ and any finite subset of $H$ have a common periodic point. 
Suppose that in Theorem 3.1 we require that $d(f(x), f(y))<d(g(x), g(y))$ for at least one $f \in H$ whenever $g(x) \neq g(y)$. We know by Corollary 2.3 above that $g$ has a fixed point $(P(g, 1) \neq \varnothing)$. A modification of the above proof and Lemma shows that under the new hypothesis the set $\{P(g, 1) \cap$ $P(h, 1): h \in H\}$ has the finite intersection property, and hence has a nonempty intersection by compactness. In fact, there is a unique(!) point $|a| \in \mid X$ such that $a=g(a)=h(a)$ for all $h \in H$.

Moreover, Theorem 3.1 is a generalization of the following theorem by Holmes, and hence of Bailey's Corollary 2 to Theorem 2 in [1].

TheOREM (Holmes [3]). Let $(X, d)$ be compact and $C$ a commutative semigroup of maps $f: X \rightarrow X$. Suppose there exist $\lambda \in(0,1)$ and $\varepsilon>0$ such that

(i) $d(x, y)<\varepsilon$ implies there exists $n(x, y) \in I^{+}$and $f_{x y} \in C$ for which $d\left(f^{n}(x), f^{n}(y)\right)<\lambda d(x, y)$. Then each finite subfamily of $C$ has a common periodic point.

Now since by definition, a semigroup is closed with respect to the given operation, we know $f^{n} \in C$ when $f \in C$ in Holmes' Theorem. Consequently, (i) can be simplified to read:

(ii) $d(x, y)<\varepsilon$ implies there exists $f_{x y} \in C$ for which $d(f(x), f(y))<$ $\lambda d(x, y)$.

Thus it is clear that Holmes' result follows from our Theorem 3.1 with $g=i$ $(i(x)=x$ for $x \in X)$ and $H=C \cup\{i\}$. The following simple example demonstrates that Theorem 3.1 indeed generalizes Holmes' result.

Example. Let $X=[0,1]$ and $d(x, y)=|x-y|$. Define $f: X \rightarrow X$ by

$$
f(x)= \begin{cases}2 x, & \text { if } 0<x<\frac{1}{2} \\ 1, & \text { if } \frac{1}{2}<x<1\end{cases}
$$

Then

$$
f^{n}(x)= \begin{cases}2^{n} x, & \text { if } 0<x<2^{-n} \\ 1, & \text { if } 2^{-n}<x<1 .\end{cases}
$$

Let $C=H=\left\{f^{n}: n \in N \cup\{0\}\right\}$, where $f^{0}=i$.

Then $d\left(f^{n}(0), f^{n}(x)\right)>d(0, x)$ for any $x \in[0,1]$ and any $f^{n} \in C$, so the hypothesis of Holmes' Theorem is not satisfied. On the other hand, let $\varepsilon=\frac{1}{4}$ and $g=f$ in the statement of our Theorem 3.1. Let $x, y \in[0,1]$ and choose notation so that $x<y$. Then if $y<\frac{1}{2}$,

$$
d(g(x), g(y))=2|x-y|>d(x, y),
$$

and we let $h=i$. If $y>\frac{1}{2}, g(y)=1$. Thus if $0<d(g(x), g(y))<\varepsilon, 0<g(x)$ $=2 x<1$ and $1-2 x<\frac{1}{4}$, so that $x>3 / 8$. Consequently, $f^{2}(x)=f^{2}(y)=$ 1 , and we have $d(g(x), g(y))>0=d\left(f^{2}(x), f^{2}(y)\right)$. In this instance let $h=$ $f^{2}$. In any event, we can choose $h$ so that the hypothesis of Theorem 3.1 is satisfied. 
4. Locally expansive maps generalized. A map $f:(X, d) \rightarrow(X, d)$ is an expansion if there exists a real $r>1$ such that $d(f(x), f(y))>r d(x, y)$. In [2] Borges considered continuous expansions on complete metric spaces, and intimated that further study of expansions is in order. Rosenholtz studied local expansions in [6] and locally expansive maps in [7]. In [7] he proved that any open locally expansive map of a connected compact metric space into itself has a fixed point. We prove:

THEOREM 4.1. Let $g$ be an open map of a compact metric space $(X, d)$ onto itself. If there exists $\varepsilon>0$ such that $0<d(x, y)<\varepsilon$ implies that for at least one $f \in C_{g}, d(f(x), f(y))<d(g(x), g(y))$, then $g$ has a periodic point.

Proof. By hypothesis, $g$ is actually a local homeomorphism of a compact metric space onto itself, and hence a covering projection. In fact (see [6]), each $a \in X$ has a neighborhood $V_{a}$ such that $g^{-1}\left(V_{a}\right)$ is the union of finitely many mutually disjoint open sets $U_{a}$ such that $\operatorname{diam}\left(U_{a}\right)<\varepsilon$, and $g\left(U_{a}\right)=$ $V_{a}$. Let $\lambda$ be the Lebesgue number of the open covering $\left\{V_{a}: a \in X\right\}$.

By Theorem 2.1 it suffices to prove:

$$
0<d(g(x), g(y))<\lambda \text { implies } d(g(x), g(y))>d(f(z), f(y))
$$

for some $z \in g^{-1}(x)$ and some $f \in C_{g}$.

To this end, suppose $0<d(g(x), g(y))<\lambda$. By the definition of $\lambda$ we can choose $a \in X$ such that $g(x), g(y) \in V_{a}$, and hence an open set $U_{a}$ such that $\operatorname{diam}\left(U_{a}\right)<\varepsilon, g\left(U_{a}\right)=V_{a}$, and $y \in U_{a}$. Since $g\left(U_{a}\right)=V_{a}, g(x)=g(z)$ for some $z \in U_{a}$. But $z, y \in U_{a}$ implies $d(z, y)<\varepsilon$; moreover, $0<d(z, y)$ since $g(x) \neq g(y)$. The hypothesis then yields $f \in C_{g}$ for which

$$
d(f(z), f(y))<d(g(z), g(y))=d(g(x), g(y)),
$$

and $(* *)$ holds.

(Compare Theorem 4.1 to Theorem 1 in [5].)

Corollary. Let $g$ be an open map of a compact metric space $(X, d)$ onto itself. If there exists $\varepsilon>0$ such that

$$
0<d(x, y)<\varepsilon \text { implies } d\left(g^{n}(x), g^{n}(y)\right)<d(g(x), g(y))
$$

for at least one nonnegative integer $n=n(x, y)$, then $g$ has a periodic point.

Note that with $n=n(x, y)=0$ in the above corollary, we obtain the result that any locally expansive open map of a compact metric space onto itself has a periodic point.

\section{REFERENCES}

1. D. F. Bailey, Some theorems on contractive mappings, J. London Math. Soc. 41 (1966), 101-106.

2. C. R. Borges, Properties of continuous expansions, Proc. Amer. Math. Soc. 50 (1975), $495-499$. 
3. R. D. Holmes, On fixed and periodic points under certain sets of mappings, Canad. Math. Bull. 12 (1969), no. 6, 813-822.

4. G. Jungck, Commuting mappings and fixed points, Amer. Math. Monthly 83 (1976), 261-263.

5. __ Periodic maps via equicontimuity, Amer. Math. Monthly 75 (1968), 265-268.

6. Ira Rosenholtz, Local expansions, derioatives, and fixed points, Fund. Math. 91 (1970), 1-4.

7. Eoidence of a conspiracy among fixed point theorems, Proc. Amer. Math. Soc. 53 (1975), 213-218.

Departmient of Mathimatics, Bradley Universtty, Pborla, IlunNots 61625 